\title{
SELETIVIDADE DE INSETICIDAS UTILIZADOS NO ALGODOEIRO SOBRE PUPAS E ADULTOS DA JOANINHA CYCLONEDA SANGUINEA (LINNAEUS, 1763)
}

\author{
E.C. Pedroso', G.A. Carvalho², M.I.S. Leite' ${ }^{2}$ D.T. Rezende ${ }^{2}$ \\ ${ }^{1}$ Universidade Federal de Lavras, CP 3037, CEP 37200-000, Lavras, MG, Brasil. E-mail: gacarval@den.ufla.br
}

\author{
RESUMO
}

Identificar pesticidas seletivos a inimigos naturais é fundamental para a boa prática do Manejo Integrado de Pragas. Este estudo objetivou avaliar a seletividade dos seguintes agroquímicos (em g i.a. $\mathrm{L}^{-1}$ de água) sobre o predador Cycloneda sanguinea (Linnaeus, 1763) (Coleoptera: Coccinellidae): triflumurom (Certero 480 SC - 0,048), espinosade (Tracer 480 SC - 0,24), clorfenapir (Pirate 240 SC - 1,2), clotianidina (Focus 500 PM - 0,33), imidaclopride+ß-ciflutrina (Connect 100/12,5 SC - 0,33/0,042), e água destilada como tratamento testemunha. As pulverizações dos produtos foram realizadas em pupas e adultos de C. sanguinea por meio de torre de Potter; as pupas permaneceram individualizadas até a emergência dos adultos quando foram formados casais. Para os adultos tratados, os casais foram formados logo após as aplicações dos produtos. Os bioensaios foram conduzidos a $25 \pm 2^{\circ} \mathrm{C}$, UR $60 \pm 10 \%$ e fotofase de $12 \mathrm{~h}$. Avaliou-se a duração, sobrevivência e razão sexual de pupas diretamente tratadas; sobrevivência e parâmetros reprodutivos de adultos diretamente tratados; sobrevivência e duração dos estádios de desenvolvimento larval e pupal, razão sexual e parâmetros reprodutivos de adultos provenientes de pupas e adultos diretamente tratados. Os inseticidas triflumuron e espinosade foram seletivos às pupas de C. sanguinea, levemente nocivos aos adultos do predador, assim como às suas fases e gerações subsequentes de desenvolvimento. Clorfenapir, clotianidina e imidaclopride+ß-ciflutrina foram tóxicos a pupas e adultos de C. sanguinea.

PALAVRAS-CHAVE: Coccinellidae, predador, avaliação de risco, pesticida, seletividade.

\section{ABSTRACT}

SELECTIVITY OF INSECTICIDES USED IN THE COTTON CROP IN REGARD TO PUPAE AND ADULTS OF THE LADYBIRD BEETLE CYCLONEDA SANGUINEA (LINNAEUS, 1763). Identifying pesticides that are selective in regard to natural enemies is critical to good practice of integrated pest management. This study was aimed to evaluate the selectivity of the following pesticides (in $\mathrm{g}$ a.i. $\mathrm{L}^{-1}$ of water) in regard to the predator Cycloneda sanguinea (Linnaeus, 1763) (Coleoptera: Coccinellidae): triflumuron (Certero SC 480 - 0.048), spinosad (Tracer 480 SC - 0.24), chlorfenapyr (Pirate SC 240 - 1.2), clothianidin (Focus 500 PM - 0.33), beta-cyfluthrin + imidacloprid (Connect 100/12, SC 5 - 0.33 / 0.042), and distilled water as a control treatment. The products were sprayed on C. sanguinea pupae and adults using a Potter's tower; the pupas were kept singly until they became adults, when couples were made. For adults, the couples were made after the application of the products. The bioassays were realized at $25 \pm 2^{\circ} \mathrm{C}, \mathrm{RU} 60 \pm 10 \%$ and photophase of $12 \mathrm{~h}$. Evaluations were made of the duration, survival and sexual rate of directly treated pupae; survival and reproductive parameters of directly treated adults; survival and duration of the larval and pupal development stages, sexual ratio and reproductive parameters of adults developed from pupae and of directly treated adults. Triflumuron and spinosad were innocuous to the $C$. sanguinea pupae, and slightly prejudicial to the adults of the predator as well to its phases and generations following development. Chlorfenapyr, chlothianidin and imidacloprid/ß-cyfluthrin were toxic to $C$. sanguinea pupae and adults.

KEY WORDS: Coccinellidae, predator, risk assessment, pesticide, selectivity.

\section{INTRODUÇÃO}

O algodoeiro é uma das mais importantes culturas agrícolas do Brasil, ocupando cerca de 1,15 milhão de hectares, com uma produção média na safra de 2007/2008 de 4,1 milhões de toneladas, sendo que desse total 2,5 milhões de toneladas é de algodão em caroço e 1,6 milhão de toneladas

${ }^{2}$ Universidade Estadual de São Paulo, Jaboticabal, SP, Brasil. 
é de pluma (COMPANHIA..., 2008). A cultura atrai e hospeda diversas pragas, as quais atacam as raízes, caules, folhas, botões florais, maçãs e capulhos. O grau de injúria na planta está diretamente ligado às densidades populacionais e/ou ao período de tempo de ocorrência dos insetos e ácaros (SANTOS, 1998). Um grande número de inimigos naturais está associado às pragas do algodoeiro, contudo a atuação e/ ou utilização desses insetos são limitadas pelas excessivas aplicações de inseticidas na lavoura. Uma das maneiras para reduzir os impactos sobre a comunidade de organismos benéficos responsáveis pelo controle biológico natural é pelo uso de inseticidas seletivos (EvANGELISTA JúNIOR et al., 2006).

Dentre as várias espécies de insetos benéficos e presentes na cultura está a joaninha Cycloneda sanguinea (Linnaeus, 1763) (Coleoptera, Coccinellidae), queé uma espécie cosmopolita pertencente à família Coccinellidae, cujos espécimes são ativos predadores de pragas. Essa espécie de joaninha está dentre os inimigos naturais mais importantes encontrados na cultura algodoeira, visto ser uma voraz predadora de pulgões que atacam a cultura. Este fato foi comprovado por estudos realizados na região de Jaboticabal, interior de São Paulo, a respeito da diversidade de inimigos naturais na cultura algodoeira, sendo que C. sanguinea correspondeu em $11 \%$ do total de inimigos naturais presentes nesse agroecossistema (SOARES et al., 1995).

BARROs et al. (2006) realizaram levantamentos populacionais dos inimigos naturais associados às pragas da cultura do algodoeiro nas safras 2001/2002 e 2002/ 2003 em Dourados, utilizando-se as variedades DeltaOpal e Fibermax 986. Verificaram que nas duas safras a família Coccinellidae foi a mais abundante do complexo de predadores, sendo que C. sanguinea foi a segunda espécie predominante com $21,15 \%$ em relação aos demais insetos benéficos, seguida de Hyppodamia convergens (GuérinMeneville, 1842) e Eriopis connexa (Germar, 1824) (Coleoptera: Coccinellidae) Em estudo realizado por RibeIRo (2007), visando realizar levantamento das espécies de predadores predominantes na cultura algodoeira, foi constatado que, na safra 2005/2006, C. sanguinea representou $55,0 \%$ dos inimigos naturais encontrados, demonstrando a existência, abundância e necessidade de se preservar esse inimigo natural para estabelecer o equilíbrio dinâmico de pragas nesse agroecossistema.

Em função da importância de $C$. sanguinea no controle natural de pragas do algodoeiro e do baixo número de informações a respeito do impacto de pesticidas usados nessa cultura sobre esse predador, realizou-se o presente trabalho com o objetivo de avaliar os efeitos de alguns compostos sobre pupas e adultos, bem como sobre a descendência da espécie.

\section{MATERIAL E MÉTODOS}

Os nomes técnicos e comerciais, a dosagem em $g$ i.a. $\mathrm{L}^{-1}$ de água e os respectivos grupos químicos dos inseticidas utilizados foram triflumurom (Certero 480 SC - 0,048; benzoilureia); espinosade (Tracer 480 SC - 0,24; spinosina); clorfenapir (Pirate 240 SC - 1,2; análogo do pirazol); clotianidina (Focus 500 PM - 0,33; neonicotinoide) e imidaclopride+ + ciflutrina (Connect 100/12,5 SC - 0,33/0,042; neonicotinoide+piretroide). No tratamento testemunha utilizou-seágua destilada. Para pulverização dosinseticidas, fez-se uso de torre de Potter à pressão de $15 \mathrm{lb} \mathrm{pol}^{-2}$, garantindo aplicação de 1,5 $\pm 0,5 \mu \mathrm{g}$ de calda $\mathrm{cm}^{-2}$, conforme recomendações da "International Organization for Biological and Integrated Control of Noxious Animals and Plants" (IOBC), visando a padronização de técnica de seletividade para inimigos naturais (SterK et al., 1999; DeGRANDE et al., 2002; van de VeIRE et al., 2002).

Efeitos dos produtos sobre pupas de C. sanguinea. Cinquenta pupas por tratamento, com aproximadamente 48 horas de idade, foram retiradas da criação de laboratório e colocadas em placas de Petri de 10 cm de diâmetro. Em seguida, receberam os produtos via pulverização em torre de Potter e foram individualizadas em placas de Petri de $5 \mathrm{~cm}$ de diâmetro que foi vedada com filme plástico de PVC. Avaliou-se a duração e sobrevivência de pupas, e razão sexual dos adultos emergidos.

Efeitos dos produtos sobre os parâmetros reprodutivos de $C$. sanguinea oriundas de pupas tratadas. Adultos recém-emergidos de pupas tratadas foram sexados e distribuídos na proporção de um casal por gaiola de PVC de $10 \mathrm{~cm}$ de diâmetro $x 10 \mathrm{~cm}$ de altura, coberta em sua extremidade superior e inferior com filme plástico fixado por meio de goma-elástica. $\mathrm{O}$ interior das gaiolas foi forrado com papel-filtro que serviu como substrato para oviposição. Ovos de Anagasta kuehniella (Zeller, 1879) (Lepidoptera: Pyralidae) e pulgões Schizaphis graminum (Rondani, 1852) (Hemiptera: Aphididae), provenientes da criação de laboratório, foram fornecidos ad libitum como presas aos casais a cada 48 horas. As posturas foram transferidas diariamente para tubos de vidro de $2,5 \mathrm{~cm}$ de diâmetro $\times 8,5 \mathrm{~cm}$ de altura, os quais foram vedados com filme plástico de PVC. Foi avaliado o período embrionário, o número total de ovos colocados durante dez dias após o período de pré-oviposição, e a viabilidade de ovos.

Efeitos dos produtos sobre larvas de primeiro a quarto instar e pupas, provenientes de pupas de C. sanguinea tratadas. Larvas de primeiro instar recém-eclodidas de ovos de adultos provenientes de pupas tratadas com os produtos químicos foram individualizadas em placas de Petri de $5 \mathrm{~cm}$ de diâmetro, contendo chumaço de algodão umedecido, 
e ovos de A. kuehniella e pulgões S. graminum, para servir-lhes dealimento. As placas foram vedadas com filme plástico de PVC. A manutenção das larvas com o fornecimento de alimento e água foi feita a cada 48 horas. Avaliou-se a sobrevivência e a duração de cada instar larval e da fase pupal, e a razão sexual dos adultos emergidos.

Efeito dos produtos na mortalidade e reprodução de adultos de $C$. sanguinea tratados. Adultos recémemergidos da criação delaboratório foram agrupados em número de 50 espécimes por tratamento, sendo 25 machos e 25 fêmeas, em placas de Petri de10 $\mathrm{cm}$ de diâmetro e submetidos a $\mathrm{CO}_{2}$ por aproximadamente dez segundos. Em seguida, receberam as pulverizações dos produtos via torre de Potter. Foram formados dez casais por tratamento, e estes mantidos em gaiolas de PVC de $10 \mathrm{~cm}$ de diâmetro $x 10 \mathrm{~cm}$ de altura, com o seu interior forrado com papel-filtro. Ovos de A. kuehniella e pulgões S. graminum, provenientes da criação de laboratório, foram fornecidos ad libitum como alimento aos casais a cada 48 horas. As posturas obtidas logo após o período de pré-oviposição foram transferidas diariamente para tubos de vidro, sendo avaliada a sobrevivência dos adultos tratados, o número total de ovos durante dez dias, o período embrionário e a viabilidade dos ovos.

Efeito dos produtos sobre larvas de primeiro a quarto instar e pupas de $C$. sanguinea oriundas de adultos tratados. As larvas recém-eclodidas, em número de cinquenta por tratamento, oriundas de ovos de adultos tratados com os compostos, foram individualizadas em placas dePetri de $5 \mathrm{~cm}$ de diâmetro contendo chumaço de algodão umedecido, ovos de A. kuehniella e pulgões S. graminum. As placas foram vedadas com filme plástico de PVC. A manutenção das larvas com o fornecimento de alimento e água foi feita a cada 48 horas. Avaliou-se a sobrevivência eo período de desenvolvimento para larvas e pupas, e a razão sexual de adultos emergidos.

Delineamento experimental. Foi utilizado o delineamento experimental inteiramente ao acaso, com cinco repetições para pupas e dez para adultos, sendo os tratamentos constituídos pelos cinco inseticidas e pela testemunha (água destilada) e cada parcela composta por dez pupas e/ou um casal de adultos.

Análise dos dados obtidos. Os dados foram submetidos à análise de variância e as médias dos tratamentos comparadas pelo teste de Tukey a 5\% de significância, por meio do programa $R$ versão 2.5.1 (R Development Core Team, 2007). O efeito total (E) de cada produto foi calculado utilizando-se a fórmula proposta por VEIRE et al. (1996), onde $\mathrm{E}=$ $100 \%$ - $(100 \%$ - Ma $) \times$ ER, em que: $\mathrm{Ma}=$ mortalidade corrigida em função do tratamento testemunha calculada pela fórmula de Abbott (Аввотт, 1925) е ER (efeito do produto no número de ovos colocados) $=$ Rt $\times \mathrm{Rc}^{-1}$ (onde Rt $=$ número médio de ovos obtidos no tratamento com o produto e Rc $=$ número médio de ovos obtidos no tratamento testemunha). Após o cálculo do efeito total, os produtos foram enquadrados em classes toxicológicas, conforme segue: classe $1=$ inócuo $(\mathrm{E}<30 \%)$, classe 2 = levemente nocivo $(30 \% \leq \mathrm{E} \leq 80 \%)$, classe $3=$ moderadamente nocivo $(80<\mathrm{E} \leq 99)$ e classe $4=$ nocivo $(\mathrm{E}>99 \%)$, de acordo com a escala proposta por membros da IOBC (VEIRE et al., 1996; HASSAN, 1997).

\section{RESULTADOS E DISCUSSÃO}

Efeitos dos produtos sobre pupas de $C$. sanguinea descendentes do predador. Os produtos testados não afetaram negativamente a duração de pupas tratadas, com médias variando de 5,09 a 5,43 dias, sendo que triflumurom e espinosade também não causaram redução de sobrevivência, com médias de $96,0 \%$ e $98,0 \%$, respectivamente. Os inseticidas clorfenapir, clotianidina eimidaclopride+ß-ciflutrina provocaram diminuiçãosignificativa de sobrevivência, apresentando médias de $72,0 \% ; 4,0 \%$ e 10,0\%, respectivamente; sendo que todos os insetos morreram logo após a emergência. A razão sexual dos espécimes oriundos de pupas tratadas com os produtos triflumurom, espinosade e clorfenapir não foi afetada, sendo de 0,$60 ; 0,70$ e 0,60 , respectivamente (Tabela 1 ).

Tabela 1 - Duração (dias) e sobrevivência de pupas (\%), e razão sexual dos adultos advindos de pupas de Cycloneda sanguinea diretamente tratadas com inseticidas. Temperatura: $25 \pm 2{ }^{\circ} \mathrm{C}$; UR: $60 \pm 10 \%$; fotofase: $12 \mathrm{~h}$.

\begin{tabular}{|c|c|c|c|}
\hline Tratamento & Duração & Sobrevivência & Razão sexual \\
\hline Testemunha & $5,12 \pm 1,09 \mathrm{a}$ & $92,0 \pm 4,91 \mathrm{a}$ & $0,63 \mathrm{a}$ \\
\hline Triflumurom & $5,40 \pm 1,30 \mathrm{a}$ & $96,0 \pm 5,03 a$ & $0,60 \mathrm{a}$ \\
\hline Espinosade & $5,24 \pm 1,11 \mathrm{a}$ & $98,0 \pm 5,33 \mathrm{a}$ & $0,70 \mathrm{a}$ \\
\hline Clorfenapir & $5,09 \pm 1,14$ a & $72,0 \pm 4,44 b$ & $0,60 \mathrm{a}$ \\
\hline Clotianidina & $5,22 \pm 1,23$ a & $4,0 \pm 0,95 \mathrm{~d}$ & * \\
\hline Imidaclopride $+ß$-ciflutrina & $5,43 \pm 1,32 \mathrm{a}$ & $10,0 \pm 1,98 \mathrm{c}$ & * \\
\hline CV $(\%)$ & 3,0 & 2,0 & 36,0 \\
\hline
\end{tabular}

Médias seguidas de mesma letra na coluna não diferem entre si pelo teste de Tukey $(a=0,05)$.

*Número de insetos insuficiente para avaliação dessa característica biológica. 
De acordo com CROFT (1990), entre os fatores que afetam a susceptibilidade de insetos a substâncias químicas está a constituição do tegumento, visto que pode apresentar, principalmente nas fases de ovo e pupa, uma camada cuticular mais impermeável dificultando a penetração dos produtos. Clorfenapir possui ação de contato e ingestão sobre insetos (WARE, 2000) e, provavelmente, seus resíduos presentes sobre os tegumentos das pupas foram os responsáveis pela alta mortalidade dos adultos oriundos delas pois, no processo de emergência, os insetos ficaram em contato direto com os tegumentos. Clotianidina e imidaclopride são compostos agonistas da acetilcolina, pertencentes ao grupo químico dos neonicotinoides, os quais causam impulsos contínuos e hiperexcitação do sistema nervoso, afetando diretamente diversos grupos de inimigos naturais (WARE; WhITACRE, 2004), fato que pode explicar a elevada toxicidade para pupas, impedindo a emergência da maioria dos adultos de C. sanguinea. Apesar de espinosade também ser agonista da acetilcolina, este não afetou a duração e sobrevivência de pupas, talvez pelo fato de que possui um sítio de ligação distinto ao dos neonicotinoides.

Trabalhos realizados por Youn et al. (2003) comprovaram que clorfenapir e imidaclopride, nas concentrações de 0,1 g i.a. $\mathrm{L}^{-1}$ e 0,05 g i.a. $\mathrm{L}^{-1}$, respectivamente, não provocaram mortalidade em pupas de Harmonia axyridis (Pallas, 1773) (Coleoptera, Coccinellidae) quando essas foram imersas em calda química durante dez segundos. Esse resultado diverge do observado no presente trabalho, provavelmente devido às maiores concentrações empregadas nos testes com pupas de $C$. sanguinea, que foram de 1,2 e 0,33 g i.a. $\mathrm{L}^{-1}$, respectivamente.

Alguns inseticidas reguladores de crescimento, como triflumurom, segundo GAZZONI (1994), são caracterizados geralmente como seletivos, e este fato ficou demonstrado neste experimento onde o produto foi inócuo para pupas de $C$. sanguinea. O período embrionário e o número total de ovos (em 10 dias após o início de oviposição) colocados por fêmeas que emergiram de pupas diretamente tratadas não foram afetados pelos produtos triflumurom e espinosade, porém a sua viabilidade sofreu redução em relação à testemunha, sendo de $80,0 \%$ para triflumurom (Tabela 2). Alguns inibidores da síntese de quitina podem impedir a síntese desse polissacarídeo no embrião, uma vez que atuam na polimerização de unidades UDP-N-acetil-D-glucosamino, o último precursor da quitina durante sua síntese (IzAwa et al., 1985). Provavelmente, isto foi a causa de mortalidade embrionária de $C$. sanguinea verificada para triflumurom.

Tabela 2 - Período embrionário (dias), número e viabilidade (\%) dos ovos colocados nos 10 primeiros dias após o início de oviposição por fêmeas de Cycloneda sanguinea provenientes de pupas diretamente tratadas com os inseticidas. Temperatura: $25 \pm 2^{\circ} \mathrm{C}$; UR: $60 \pm 10 \%$; fotofase: $12 \mathrm{~h}$.

\begin{tabular}{lccc}
\hline Tratamento & Período embrionário & Número de ovos & Viabilidade \\
\hline Testemunha & $2,89 \pm 0,45 \mathrm{a}$ & $177,60 \pm 11,65 \mathrm{a}$ & $89,0 \pm 7,03 \mathrm{a}$ \\
Triflumurom & $2,93 \pm 0,47 \mathrm{a}$ & $136,20 \pm 12,33 \mathrm{a}$ & $80,0 \pm 4,71 \mathrm{~b}$ \\
Espinosade & $2,78 \pm 0,43 \mathrm{a}$ & $133,00 \pm 13,71 \mathrm{a}$ & $85,0 \pm 4,85 \mathrm{a}$ \\
\hline CV $(\%)$ & 6,0 & & 4,0 \\
\hline
\end{tabular}

Médias seguidas de mesma letra na coluna não diferem entre si pelo teste de Tukey $(a=0,05)$.

Tabela 3 - Duração (dias) e sobrevivência (\%) de larvas de Cycloneda sanguinea oriundas de adultos advindos de pupas diretamente tratadas com os inseticidas. Temperatura: $25 \pm 2^{\circ} \mathrm{C}$; UR: $60 \pm 10 \%$; fotofase: $12 \mathrm{~h}$.

\begin{tabular}{|c|c|c|c|c|}
\hline \multirow{2}{*}{ Tratamento } & \multicolumn{2}{|c|}{ Primeiro instar } & \multicolumn{2}{|c|}{ Segundo instar } \\
\hline & Duração & Sobrevivência & Duração & Sobrevivência \\
\hline Testemunha & $2,16 \pm 0,57 \mathrm{a}$ & $100,0 \pm 0,00 \mathrm{a}$ & $2,38 \pm 0,62 \mathrm{a}$ & $100,0 \pm 0,00 \mathrm{a}$ \\
\hline Triflumurom & $2,18 \pm 0,63 \mathrm{a}$ & $100,0 \pm 0,00 \mathrm{a}$ & $2,90 \pm 0,91 \mathrm{a}$ & $100,0 \pm 0,00 \mathrm{a}$ \\
\hline Espinosade & $2,80 \pm 0,79 \mathrm{~b}$ & $100,0 \pm 0,00 \mathrm{a}$ & $2,56 \pm 0,55 \mathrm{a}$ & $100,0 \pm 0,00 \mathrm{a}$ \\
\hline CV $(\%)$ & 9,0 & - & 19,0 & - \\
\hline \multirow{2}{*}{ Tratamento } & \multicolumn{2}{|c|}{ Terceiro instar } & \multicolumn{2}{|c|}{ Quarto instar } \\
\hline & Duração & Sobrevivência & Duração & Sobrevivência \\
\hline Testemunha & $2,06 \pm 0,49 a$ & $100,0 \pm 0,00 \mathrm{a}$ & $2,7 \pm 0,73 \mathrm{a}$ & $100,0 \pm 0,00 \mathrm{a}$ \\
\hline Triflumurom & $2,08 \pm 0,52 \mathrm{a}$ & $100,0 \pm 0,00 \mathrm{a}$ & $3,04 \pm 0,71 \mathrm{a}$ & $100,0 \pm 0,00 \mathrm{a}$ \\
\hline Espinosade & $2,24 \pm 0,51 \mathrm{a}$ & $100,0 \pm 0,00 \mathrm{a}$ & $2,78 \pm 0,69 \mathrm{a}$ & $100,0 \pm 0,00 \mathrm{a}$ \\
\hline CV $(\%)$ & 9,0 & - & 13,0 & - \\
\hline
\end{tabular}

Médias seguidas de mesma letra na coluna não diferem entre si pelo teste de Tukey $(\alpha=0,05)$. 
A duração e sobrevivência de larvas do predador provenientes de adultos advindos de pupas diretamente tratadas sofreram influência apenas de espinosade, que provocou o aumento da duração do primeiro instar para 2,8 dias (Tabela 3). A não interferência de triflumurom sobre a duração e sobrevivência das larvas ocorreu possivelmente devido à metabolização e/ ou excreção do produto pelo seu organismo. Cosme et al. (2007) avaliaram o efeito de inseticidas naturais e sintéticos sobre ovos e larvas de $C$. sanguinea e concluíram que teflubenzurom, pertencente ao mesmo grupo químico de triflumurom, não reduziu a duração dos estádios larvais quando larvas de primeiro instar foram tratadas. Os dados encontrados na presente pesquisa (Tabela 3) corroboram os resultados relatados por esses autores. Levando-se em consideração o efeito total (E) dos tratamentos, triflumurom e espinosade foram inócuos (classe 1) para pupas e clorfenapir, clotianidina e imidaclopride $+b$-ciflutrina foram nocivos (classe 4) (Tabela 4).
Efeitos dos produtos sobre adultos de C. sanguinea diretamente tratados e na sua descendência. Triflumurom eespinosade permitiram a sobrevivência de $100,0 \%$ dos adultos, e clorfenapir possibilitou 60,0\% de sobrevivência, mas os espécimes morreram todos após $24 \mathrm{~h}$ das pulverizações. Clotianidina e imidaclopride $+\beta$-ciflutrina não permitiram a sobrevivência dos insetos logo após as aplicações (Tabela 5).

Grafton-Cardwell; Gu (2003) expuseram o predador Rodolia cardinalis (Mulsant) (Coleoptera, Coccinellidae) durante 72 horas, a folhas de citros tratadas com espinosade (105 gi.a. ha ${ }^{-1}$ ) eobservaram que $97,8 \%$ dos espécimes sobreviveram, comprovando também a inocuidade desse inseticida para essa espécie. Testes realizados por Lo (2004) com o cocinelídeo Halmus chalybeus (Boisduval, 1835) (Coleopetra, Coccinellidae) comprovaram que buprofezina $\left(0,125\right.$ gi.a. $\left.\mathrm{L}^{-1}\right)$, produto do mesmo grupo químico do triflumurom, não se apresentou tóxico aos adultos do predador quando estes foram imersos durante 10 segundos em calda química.

Tabela 4 - Mortalidade (\%), número de ovos até o décimo dia após o início de oviposição, efeito total (E) (\%) e classificação dos produtos em função da escala de toxicidade da IOBC, de espécimes oriundos de pupas de Cycloneda sanguinea diretamente tratadas com os inseticidas. Temperatura: $25 \pm 2{ }^{\circ} \mathrm{C}$; UR: $60 \pm 10 \%$; fotofase: $12 \mathrm{~h}$.

\begin{tabular}{lcccc}
\hline Tratamentos & $\mathrm{Ma}(\%)^{1}$ & № total de ovos & $\mathrm{E}(\%)^{2}$ & Classe $^{3}$ \\
\hline Testemunha & - & 176,40 & - & - \\
Triflumurom & 0,00 & 126,60 & 25,00 & 1 \\
Espinosade & 0,00 & 126,30 & 24,00 & 1 \\
Clorfenapir & 22,00 & $*$ & 100,00 & 4 \\
Clotianidina & 100,00 & $*$ & 100,00 & 4 \\
Imidaclopride+ß-ciflutrina & 100,00 & $*$ & 100,00 & 4 \\
\hline
\end{tabular}

${ }^{1}$ Mortalidade no tratamento corrigida pela fórmula de Abbott (Аввотт, 1925).

${ }^{2}$ Efeito total do produto sobre o predador.

${ }^{3}$ Classe de toxicidade segundo a IOBC.

*Número de insetos insuficiente para avaliação dessa característica biológica.

Tabela 5 - Sobrevivência (\%) de adultos diretamente tratados com os inseticidas após 24 h da aplicação, período embrionário (dias), número total e viabilidade de ovos (\%) de Cycloneda sanguinea colocados em 10 dias após a aplicação dos inseticidas. Temperatura: $25 \pm 2{ }^{\circ} \mathrm{C}$; UR: $60 \pm 10 \%$; fotofase: $12 \mathrm{~h}$.

\begin{tabular}{lcccc}
\hline Tratamento & Sobrevivência & $\begin{array}{c}\text { Período } \\
\text { embrionário }\end{array}$ & № Total de ovos & Viabilidade \\
\hline Testemunha & $100,0 \pm 0,00 \mathrm{a}$ & $2,98 \pm 0,87 \mathrm{a}$ & $163,90 \pm 11,87 \mathrm{a}$ & $88,35 \pm 6,45 \mathrm{a}$ \\
Triflumurom & $100,0 \pm 0,00 \mathrm{a}$ & $2,93 \pm 0,77 \mathrm{a}$ & $106,90 \pm 10,56 \mathrm{~b}$ & $81,77 \pm 5,55 \mathrm{a}$ \\
Espinosade & $100,0 \pm 0,00 \mathrm{a}$ & $2,92 \pm 0,69 \mathrm{a}$ & $108,70 \pm 10,62 \mathrm{~b}$ & $79,66 \pm 6,79 \mathrm{a}$ \\
Clorfenapir & $60,0 \pm 8,21 \mathrm{~b}$ & $*$ & $*$ & $*$ \\
Clotianidina & $0,0 \pm 0,00 \mathrm{c}$ & $*$ & $*$ & $*$ \\
Imidaclopride+ß-ciflutrina & $0,0 \pm 0,00 \mathrm{c}$ & $*$ & 37,0 & 6,0 \\
\hline CV $(\%)$ & 14,3 & 2,0 & $*$
\end{tabular}

Médias seguidas de mesma letra na coluna não diferem entre si pelo teste de Tukey $(a=0,05)$.

*Número de insetos insuficiente para avaliação dessa característica biológica. 
Resultados semelhantes aos do presente estudo foram obtidos por CLOYD; DicKINSON (2006), onde a ação direta de neonicotinoides sobre a joaninha Cryptolaemus montrouzieri (Mulsant, 1853) (Coleoptera, Coccinellidae) foi estudada. Os resultados evidenciaram mortalidade elevada $24 \mathrm{~h}$ após a aplicação dos compostos, sendo que, decorridas $48 \mathrm{~h}$, a mortalidade atingiu 100,0\% para o tratamento à base de clotianidina. A permetrina pertencente ao mesmo grupoquímico da ß-ciflutrina (grupo químico dos piretroides), causou 100,0\% de mortalidade de adultos de $H$. chalybeus quando estes foram imersos em calda química durante 10 segundos (Lo, 2004).

O período embrionário e a viabilidade de ovos de adultos diretamente tratados não foram afetados por triflumurom e espinosade, porém, reduziram o número total de ovos colocados nos 10 primeiros dias após o início de oviposição, com médias de 106,9 e 108,7, respectivamente (Tabela 5).

Existem poucos trabalhos em literatura sobre o impacto de espinosade sobre inimigos naturais, incluindo C. sanguinea. Entretanto, MAHDIAN et al.
(2007) observaram que o número de ovos de Picromerus bidens (Hemiptera: Pentatomidae) sofreu redução de $12,0 \%$ quando os casais foram expostos por $48 \mathrm{~h}$ em contato com superfícies contaminadas com esse compostos na dosagem de 0,096 g i.a. $\mathrm{L}^{-1}$. Triflumurom e espinosade não interferiram na duração e sobrevivência de larvas de primeiro, segundo, terceiroe quarto instares de C. sanguinea provenientes de adultos diretamente tratados com os compostos, apresentando médias que variaram de 2,0 a 3,0 dias e de $100,00 \%$, respectivamente (Tabela 6).

Quanto ao efeito total (E) dos inseticidas, triflumurom e espinosade foram levemente nocivos (classe 2) para adultos de C. sanguinea, e clorfenapir, clotianidina e imidaclopride+ß-ciflutrina foram nocivos (classe 4) (Tabela 7). São escassos os estudos que abordam de forma mais abrangente os efeitos de inseticidas e seus reflexos sobre as gerações desse predador; desta forma, se fazem necessárias novas pesquisas para averiguar os efeitos de novos produtos nas fases subsequentes (descendência) de C. sanguinea.

Tabela 6 - Duração (dias) e sobrevivência (\%) de larvas de Cycloneda sanguinea oriundas de adultos diretamente tratados com os inseticidas. Temperatura: $25 \pm 2{ }^{\circ} \mathrm{C}$; UR: $60 \pm 10 \%$; fotofase: $12 \mathrm{~h}$.

\begin{tabular}{|c|c|c|c|c|}
\hline \multirow{2}{*}{ Tratamento } & \multicolumn{2}{|c|}{ Primeiro instar } & \multicolumn{2}{|c|}{ Segundo instar } \\
\hline & Duração & Sobrevivência & Duração & Sobrevivência \\
\hline Testemunha & $2,18 \pm 0,33 \mathrm{a}$ & $100,0 \pm 0,00 \mathrm{a}$ & $2,48 \pm 0,49 a$ & $100,0 \pm 0,00 \mathrm{a}$ \\
\hline Triflumurom & $2,10 \pm 0,41 \mathrm{a}$ & $100,0 \pm 0,00 \mathrm{a}$ & $2,72 \pm 0,51 \mathrm{a}$ & $100,0 \pm 0,00 \mathrm{a}$ \\
\hline Espinosade & $2,00 \pm 0,39 \mathrm{a}$ & $100,0 \pm 0,00 \mathrm{a}$ & $2,84 \pm 0,53 \mathrm{a}$ & $100,0 \pm 0,00 \mathrm{a}$ \\
\hline CV (\%) & 4,0 & - & 10,0 & - \\
\hline \multirow{2}{*}{ Tratamento } & \multicolumn{2}{|c|}{ Terceiro instar } & \multicolumn{2}{|c|}{ Quarto instar } \\
\hline & Duração & Sobrevivência & Duração & Sobrevivência \\
\hline Testemunha & $2,24 \pm 0,43 \mathrm{a}$ & $100,0 \pm 0,00 \mathrm{a}$ & $3,00 \pm 0,65 a$ & $100,0 \pm 0,00 \mathrm{a}$ \\
\hline Triflumurom & $2,62 \pm 0,57 \mathrm{a}$ & $100,0 \pm 0,00 \mathrm{a}$ & $2,76 \pm 0,55 a$ & $100,0 \pm 0,00 \mathrm{a}$ \\
\hline Espinosade & $2,10 \pm 0,48 \mathrm{a}$ & $100,0 \pm 0,00 \mathrm{a}$ & $2,90 \pm 0,61 \mathrm{a}$ & $100,0 \pm 0,00 \mathrm{a}$ \\
\hline $\mathrm{CV}(\%)$ & 15,0 & - & 5,0 & - \\
\hline
\end{tabular}

Médias seguidas de mesma letra na coluna não diferem entre si pelo teste de Tukey $(\alpha=0,05)$.

Tabela 7 - Mortalidade (\%), número de ovos até o décimo dia após o início de oviposição, efeito total (E) (\%) e classificação dos produtos em função da escala de toxicidade proposta pela IOBC, de espécimes oriundos de adultos de Cycloneda sanguinea tratados com os inseticidas. Temperatura: $25 \pm 2{ }^{\circ} \mathrm{C}$; UR: $60 \pm 10 \%$; fotofase: $12 \mathrm{~h}$.

\begin{tabular}{lcccc}
\hline Tratamento & $\mathrm{Ma}(\%)^{1}$ & № total de ovos & $\mathrm{E}(\%)^{2}$ & Classe $^{3}$ \\
\hline Testemunha & - & 155,70 & - & - \\
Triflumurom & 0,00 & 98,40 & 37,00 & 2 \\
Espinosade & 0,00 & 94,70 & 39,00 & 2 \\
Clorfenapir & 100,00 & $*$ & 100,00 & 4 \\
Clotianidina & 100,00 & $*$ & 100,00 & 4 \\
Imidaclopride+ß-ciflutrina & 100,00 & $*$ & 100,00 & 4 \\
\hline
\end{tabular}

${ }^{1}$ Mortalidade no tratamento corrigida pela fórmula de Abbott (Аввотт, 1925).

${ }^{2}$ Efeito total do produto sobre o predador.

${ }^{3}$ Classe de toxicidade segundo a IOBC.

*Número de insetos insuficiente para avaliação dessa característica biológica. 


\section{CONCLUSÕES}

Os inseticidas triflumurom e espinosade são seletivos para pupas de C. sanguinea, assim como às fases subsequentes de desenvolvimento do predador. Triflumurom e espinosade são levemente nocivos para adultos do predador e também às suas subsequentes fases de desenvolvimento. Clorfenapir, clotianidina e imidaclopride+\$-ciflutrina são tóxicos para pupas eadultos de C. sanguinea, necessitando de novos estudos em condições de casa de vegetação e campo para comprovação ou não de sua toxicidade.

\section{AGRADECIMENTOS}

À FAPEMIG; CAPES e ao CNPq pelo financiamento do presente trabalho.

\section{REFERÊNCIAS}

ABBOTT, W.S. A method of computing the effectiveness of an insecticide. Journal of Economic Entomology, v.18, p.265-267, 1925.

BARROS, R.; DEGRANDE, P.E.; RIBEIRO, J.F.; RODRIGUES, A.L.L.; NOGUEIRA, R.F.; FERNANDES, M.G. Flutuação populacional de insetos predadores associados a pragas do algodoeiro. Arquivos do Instituto Biológico, São Paulo, v.73, n.1, p.57-64, 2006.

CLOYD, R.A.; DICKINSON, A. Effect of insecticides on mealybug destroyer (Coleoptera, Coccinellidae) and parasitoid Leptomastix dactylopii (Hymenoptera, Encyrtidae), natural enemies of citrus mealybug (Homoptera, Pseudococcidae). Journal of Economic Entomology, v.99, n.5, p.1596-1604, 2006.

\section{COMPANHIA NACIONAL DE ABASTECIMENTO.} (Brasil). Acompanhamento da safra brasileira de grãos - safra 2007/2008. Quarto levantamento - janeiro, 2008. Disponível em: <http://www.conab.gov.br/ conabweb/download/safra/estudo_safra.pdf.> Acesso em: 18 jan. 2008.

COSME, L.V.; CARVALHO, G.A.; MOURA, A.P. Efeitos de inseticidas botânico e sintéticos sobre ovos e larvas de Cycloneda sanguinea (Linnaeus) (Coleoptera: Coccinellidae) em condições de laboratório. Arquivos do Instituto Biológico, São Paulo, v.74, n.3, p.251-258, 2007.

CROFT, B.A. Arthropod biological control agents and pesticides. New York: Wiley-Interscience, 1990. 723p. (Environmental Science and Technology)

DEGRANDE, P.E.; REIS, P.R.; CARVALHO, G.A.; BELARMINO, L.C. Metodologia para avaliar o impacto de pesticidas sobre inimigos naturais. In: PARRA, J.R.P.;
BOTELHO, P.S.M.; CORRÊA-FERREIRA; BENTO, J.M.S. (Ed.). Controle biológico no Brasil: parasitóides e predadores. São Paulo: Manole, 2002. p.71-94.

EVANGELISTA JÚNIOR, W.S.; ZANUNCIO JÚNIOR, J.S.; ZANUNCIO, J.C. Controle biológico de artrópodes pragas do algodoeiro com predadores e parasitóides. Revista Brasileira de Oleaginosas e Fibrosas, v.10, n.3, p.1147-1165, 2006.

GAZZONI, D.L. Pesquisa em seletividade de inseticidas no Brasil: uma abordagem conceitual e metodológica. In: SIMPÓSIO DE CONTROLE BIOLÓGICO, 4., 1994, Pelotas. Anais. Pelotas, RS: 1994. p.119-124.

GRAFTON-CARDWELL, E.E.; GU, P. Conserving vedalia beetle, Rodolia cardinalis (Mulsant) (Coleoptera, Coccinellidae), in Citrus: a continuing challenge as new insecticides gain registration. Journal of Economic Entomology, v.96, n.5, p.1388-1398, 2003.

HASSAN, S.A. Métodos padronizados para testes de seletividade, com ênfase em Trichogramma. In: PARRA. J.R.P.; ZUCCHI, R.A. (Ed.). Trichogramma e o controle biológico aplicado. Piracicaba: FEALQ, 1997. p.207-233.

HASSAN, S.A.; DEGRANDE, P.E. Methods to test the side effects of pesticides on Trichogramma. In: PARRA, J.R.P; ZUCCHI, R.A. (Ed.). Curso de controle biológico com Trichogramma. Piracicaba: FEALQ, 1996. p.63-74.

IZAWA, Y.; UCHIDA, M.; SUGIMOTO, T.; ASAL, T. Inhibition of chitin biosynthesis by buprofezin analogs in relation to their activity controlling Nilaparvata lugens Stal. Pesticide Biochemistry and Physiology, v.24, n.4, p.343-347, 1985.

LO, P.L. Toxicity of pesticides to Halmus chalybeus (Coleoptera: Coccinellidae) and the effect of three fungicides on their densities in a citrus orchard. New Zealand Journal of Crop and Horticultural Science, v.32, n.1, p.69-76, 2004.

MAHDIAN, K.; VAN LEEUWEN, T.; TIRRY, L.; CLERCQ, P. Susceptibility of the predatory stinkbug Picromerus bidens to selected insecticides. Journal of the International Organization for Biological Control, v. 52, n.6, p.765-774, 2007.

RIBEIRO, J.F. Seletividade de inseticidas aos artrópodes-predadores de pragas do algodoeiro em condições de campo. 2007. 36p. Dissertação (Mestrado em Agronomia) - Curso de Pós-graduação em Produção Vegetal, Universidade Federal da Grande Dourados, Dourados, 2007.

R DEVELOPMENT CORE TEAM: R: a language and environment for statistical computing. Vienna, Austria: R Foundation for Statistical Computing, 2007.

SANTOS, W.J. dos Problemas e soluções do manejo integrado de pragas do algodão. In: SEMINÁRIO 
ESTADUAL DO ALGODÃO, 4., 1998, Cuiabá. Anais. Cuiabá: Fundação MT, 1998. p.39-48.

SOARES, J.J.; BRAZ, B.A.; BUSOLI, A.C. Impacto de herbicidas sobre artrópodes benéficos associados ao algodoeiro. Pesquisa Agropecuária Brasileira, v.30, n.9, p.1135-1140, 1995.

STERK, G.; HASSAN, S.A.; BAILLOD, M.; BAKKER, F.; BIGLER, F.; BLÜMEL, S.; BOGENSCHÜTZ, H.; BOLLER, E.; BROMAND, B.; BRUN, J.; CALLIS, J.N.M.; COREMANS-PELSENEER, J.; DUSO, C.; GARRIDO, A.; GROVE, A.; HEIMBACH, U.; HOKKANEN, H.; JACAS, J.; LEWIS, G.; MORETH, L.; POLGAR, L.; ROVERSTI, L.; SAMSØE-PETERSEN, L.; SAUPHANOR, B.; SCHAUB, L.; STÄUBLI, A.; TUSET, J.J.; VAINIO, A.; van de VEIRE, M.; VIGGIANI, G.; VIÑUELA, E.; VOGT, H. Results of the seventh joint pesticide testing programme carried out by the IOBC/WPRS-Working Group 'Pesticides and Beneficial Organisms'. BioControl, v.44, n.1, p.99-117, 1999.

VAN DE VEIRE, M.; STERK, G.; VAN DER STAAIJ, M.; RAMAKERS, P.M.J.; TIRRY, L. Sequential testing scheme for the assessment of the side-effects of plant protection products on the predatory bug Orius laevigatus. BioControl, v.47, n.1, p.101-113, 2002.

VEIRE, M.; SMAGGHE, G.; DEGHEELE, D. A laboratory test method to evalue the effect of 31 pesticides on the predatory bug, Orius laevigatus (Heteroptera, Anthocoridae). Entomaphaga, v.41, n.2, p.235-243, 1996.

WARE, G.W. An introduction to insecticides. 2000. Disponível em: <http://ipmworld.umn.edu/chapters/ware. htm>. Acesso em: 4 dez. 2007.

WARE, G.W.; WHITACRE, D.M. An introduction to insecticides. 2004. Disponível em: <http:/ /ipmworld.umn. edu/chapters/ware.htm>. Acesso em: 4 dez. 2007.

YOUN, Y.N.; SEO, M.J.; SHIN, J.G.; JANG, C.; YU, Y.M. Toxicity of greenhouse pesticides to multicolored Asian lady beetles, Harmonia axyridis (Coleoptera: Coccinellidae), Biological Control, v.28, n.2, p.164-170, 2003.

Recebido em: 12/11/10

Aceito em 1/11/11 